Como citar este artigo: Sulzbacher MM, Santos AB, Basso RDB, Hirsh CE, Ludwig MS, Heck TC. Efeitos do tratamento com glutamina via enteral em modelo animal de sepse. Revista Saúde

(Sta. Maria). 2018; 44(2).

Autor correspondente: Maicon Machado Sulzbacher Universidade Regional do Noroeste do Estado do Rio Grande do Sul (UNIJUI), ljuí, RS, Brasil. (endereço)

Pais: Brasil

Resumo da Biografia

Formado em Enfermagem,

mestrado em andamento no

Programa de Pós Graduação

em Atenção Integral a Saúde

(PPCAIS), Departamento

Ciências da Vida (DCVida), pela Universidade Regional do Noroeste do Estado do Rio Grande do Sul (UNIJUÍ) ljuí, Universidade de Cruz Alta (UNICRUZ) Cruz Alta, RS, Brasil. RS, Brasil.

Data de Submissão: 19/02/18

Data de aceite: 18/07/2018

Conflito de Interesse: Não há conflito de interesse

\section{Efeitos do tratamento com glutamina via enteral em modelo animal de sepse}

\section{Effects of enteral glutamine treatment on animal model of sepsis}

\author{
Maicon Machado Sulzbacher, Analú Bender Santos, Renan Daniel Bueno \\ Basso, Gabriela Elisa Hirsh, Mirna Stela Ludwig, Thiago Gomes Heck
}

\section{RESUMO}

Objetivo: Avaliar o tratamento enteral com L-glutamina nos parâmetros clínicos em modelo experimental de sepse. Métodos: Foram utilizados 24 camundongos B6129SF2/J: Primeiramente, 11 camundongos foram divididos em três grupos para avaliar o modelo experimental que mimetize a fase hipodinâmica na sepse. Os animais receberam solução fisiológica ou solução fecal a $10 \%$ ou $20 \%$ (i.p.), sendo avaliados glicemia e temperatura corporal em $24 \mathrm{~h}$. Avaliamos o efeito do tratamento de L-glutamina por 47h na glicemia, temperatura retal, contagem de leucócitos e peso corporal em 13 animais nos grupos: Controle, Sepse, Glutamina e Sepse+Glutamina. Resultados: A solução fecal $20 \%$ induziu a sepse, evidenciando hipotermia, hipoglicemia e leucopenia. $O$ tratamento com L-glutamina evitou a leucopenia. Conclusão: 0 tratamento com L-glutamina previne a leucopenia no modelo experimental de sepse, sem melhorar outros parâmetros clínicos. Sendo a resposta imune essencial na sepse, o uso de glutamina pode ser útil como terapia adjuvante na sepse.

Palavras Chave: Sepse; Modelos Animais; Glutamina; Leucopenia.

\section{ABSTRACT}

The aim of study was to evaluate the effects of enteral glutamine treatment on clinical parameters in an animal model of sepsis. Were used 24 mice (B6129SF2/J) in two experiments: First 11 mice were divided into three groups to evaluate the animal model better to simulate the sepsis. Animals received saline (control) or faecal solution 10 or $20 \%$ (i.p), and were evaluated glycaemia and rectal temperature for $24 \mathrm{~h}$. Second 13 mice were divided into: Control, Sepsis, Glutamine and Sepsis+Glutamine. Glutamine was administered by gavage $(1 \mathrm{~g} / \mathrm{kg})$ each $4 \mathrm{~h}$ for $47 \mathrm{~h}$. Glycaemia, rectal temperature, leukocyte count and body weight was evaluated. Results: The administration of $20 \%$ faecal solution induced hypodynamic phase of sepsis with hypothermia, hypoglycaemia and low leukocyte count. The treatment with glutamine avoids the decrease in leukocyte. Conclusion: Since the immune response is essential in sepsis, the use of glutamine can be useful as adjuvant therapy in sepsis.

Keywords: Sepsis; Animal Models; Glutamine; Leukopenia. 


\section{Introdução}

A Anualmente, ocorrem 31,5 milhões de casos de sepse no mundo, resultando em uma mortalidade de 5,3 milhões de pacientes1, com elevados custos decorrentes das internações em hospitais2. A sepse é um processo infeccioso sistêmico gera uma resposta inflamatória e disfunção imunológica, com uma resposta inicial exacerbada (fase "hiperdinâmica"), que evolui para alteração em parâmetros clínicos, como a leucopenia, caracterizando imunodepressão3, principal preditor de mortalidade nesta doença4. Além disso, observa-se hipotermia5, hipoglicemia6 e perda de massa muscular7, caracterizando a fase "hipodinâmica" da doença3, que frequentemente evolui para o choque séptico e morte8.

O quadro hipercatabólico presente na sepse e a severa redução da massa muscular7, sugerem que uma suplementação com aminoácidos/proteínas poderia auxiliar no seu tratamento9. A suplementação com nutrição por via enteral, favorece o controle sobre o metabolismo energético, e evita isquemia intestinal e a disseminação dos patógenos para corrente sanguínea10, o que a torna uma boa alternativa para o fornecimento de substrato energético e para a adequação às metas calóricas e proteicas do organismo, além de evitar complicações pela diminuição do tempo de internação hospitalar11. 0 aminoácido glutamina destaca-se por sua função anabólica e estimuladora do sistema imune12. A diminuição dos seus níveis plasmáticos na sepse, está correlacionada com maior mortalidade13. Esses fatores sugerem, portanto, que a suplementação com este aminoácido possa ser alternativa no tratamento da doença14.

Existem alguns modelos experimentais de sepse que buscam mimetizar o foco infeccioso com infecção do trato abdominal. Ainda, embora existam alguns modelos experimentais de indução da sepse estabelecidos na literatura, como o da peritonite induzida por meio de cirurgia com a ligação e perfuração do ceco (CLP) 15 , ou pela administração intraperitoneal de lipopolissacarídeo (LPS)16, nenhum deles demonstra a fase hipodinâmica da doença, nem mimetiza a infecção com bactérias comensais, além de apresentar a desvantagem da realização de intervenção cirúrgica, o que mostra a importância da realização de mais estudos nesse sentido.

Neste contexto, este trabalho buscou adequar o modelo experimental de peritonite $10 \%$, para um modelo de infecção sistêmica/sepse e avaliar o efeito do tratamento enteral com glutamina sobre os parâmetros clínicos da fase hipodinâmica da sepse.

\section{Métodos}

\section{Animais}

Foram utilizados 24 camundongos da linhagem B6129SF2/J (B6) com 90 dias de idade e $30 \pm 2$ g, provenientes do biotério da Universidade Regional do Noroeste do Estado do Rio Grande do Sul (UNIJUÍ), mantidos em gaiolas semi-metabólicas, ambiente com temperatura controlada $\left(22 \pm 2^{\circ} \mathrm{C}\right)$ e ciclo claro/escuro de 12/12 horas. Os animais receberam ração padronizada para animais de laboratório (Nuvilab CR-1) e água ad libitum. Todos os experimentos foram realizados no Laboratório de Ensaios Biológicos da UNIJUÍ (LEBio) e os protocolos experimentais aprovados pela Comissão de Ética no Uso de Animais (CEUA) da UNIJUÍ sob o parecer n48/2016.

\section{Desenho Experimental}

Os animais foram divididos em Experimento 1: avaliação dose-resposta e Experimento 2: avaliação dos efeitos do tratamento com L-glutamina.

No Experimento 1 onze $(n=11)$ camundongos foram divididos em três grupos: Animais submetidos à administração intraperitoneal de solução fecal na concentração de 10\% (grupo SEP 10\%, n= 5) e $20 \%$ (grupo SEP 20\%, $n=3$ ) e grupo 
Controle (CONT, $n=3$ ). Os animais dos grupos com sepse receberam administração de solução fecal $10 \%$ e $20 \%$ via intraperitoneal e os animais do grupo controle, solução fisiológica 0,9\% em uma dose de $5 \mu \mathrm{L} / \mathrm{g}$ de peso corporal17. Neste experimento foram mensuradas a glicemia e a temperatura corporal antes da administração intraperitoneal $(\mathrm{T}=0)$, 4 ( $\mathrm{T}=4), 8$ ( $\mathrm{T}=8), 12$ ( $\mathrm{T}=12)$ e 24 horas ( $\mathrm{T}=24)$ após.

No experimento 2, foi avaliado o efeito do tratamento da sepse com L-glutamina, sendo treze animais $(n=13)$ divididos em quatro grupos; controle (CONT, $n=4$ ), sepse (SEPSE, $n=3$ ), glutamina (GLN n= 3) e sepse + glutamina (SEPSE+GLN, $n=3)$. Os animais do grupo sepse receberam administração intraperitoneal de solução fecal $20 \%$ em uma dose de $5 \mu \mathrm{L} / \mathrm{g}$ de peso corporal, enquanto os animais do grupo controle receberam solução fisiológica 0,9\% na mesma dose17. Foi mensurado o peso corporal antes e 48 horas após o início do protocolo experimental de sepse. A glicemia e temperatura corporal foram aferidas antes da submissão à sepse ( $\mathrm{T}=0)$, e 1 ( $\mathrm{T}=1), 4(\mathrm{~T}=4), 8$ ( $\mathrm{T}=8), 12$ ( $\mathrm{T}=12), 24$ ( $\mathrm{T}=24), 36$ ( $\mathrm{T}=36)$ e 48 horas ( $T=48)$ após. Ao final do experimento os animais foram mortos por decapitação para a coleta do sangue, no qual foi realizada a contagem de leucócitos totais.

\section{Preparo da solução fecal para indução da sepse}

A solução fecal nas concentrações de $10 \%$ e $20 \%$ foi preparada com fezes frescas dos respectivos animais. Após coleta das fezes, se fez um homogeneizado das mesmas em solução fisiológica $0,9 \%$, de modo a obtermos uma concentração fecal de 10\% e 20\%. Em seguida, este homogeneizado foi filtrado através de gaze estéril para que fossem eliminadas as partículas maiores e, posteriormente, centrifugado a $1000 \mathrm{~g}$ por 3 segundos.

\section{Intervenção com L-glutamina}

Os grupos GLN e SEPSE+GLN foram suplementados com L-glutamina $(1 \mathrm{~g} / \mathrm{Kg} /$ dia por gavagem orogástrica, 3 vezes por dia, com intervalos de 4 horas, iniciando-se 1 hora após a indução da sepse, totalizando 47 horas de tratamento com glutamina ${ }^{16}$.

\section{Avaliação de parâmetros clínicos após indução de sepse}

Os animais foram pesados antes e ao final do protocolo experimental em balança digital. A glicemia foi mensurada através de punção venosa na parte distal da cauda dos animais através de glicosímetro (Optium Execeed) e a temperatura foi mensurada com a inserção retal de termômetro (Techline).

Após eutanásia, o tecido esplênico dos animais foi pesado em balança analítica e aproximadamente $20 \mu \mathrm{L}$ de sangue total foi coletado em microtubos contendo $400 \mu \mathrm{L}$ de líquido de Turk (corante de Turk RenyLab ${ }^{\circledR}$ utilizado para a contagem de leucócitos totais que consiste em $1 \%$ de Solução de Violeta Genciana em Solução de ácido acético 2\%). Para a contagem de leucócitos totais foi utilizado $20 \mu \mathrm{L}$ de sangue homogeneizado em um microtubo com $0,4 \mathrm{~mL}$ de líquido de Turk. O microtubo foi agitado para induzir a hemólise e a contagem de leucócitos foi realizada por microscopia (aumento de 400x) em câmara de Neubauer, considerando toda a área reticulada dos 4 cantos da câmara. 0 número de células foi definido pela soma do número de células nos quatro quadrantes e multiplicado por 50 .

\section{Análise estatística}

Os resultados foram expressos como média \pm desvio padrão. Para comparação entre os diferentes grupos de tratamento, no mesmo tempo experimental, foi realizado teste não paramétrico de Kruskal-Wallis, seguido de pós-teste de Dunns. Já para comparação entre os diferentes tempos no mesmo grupo de tratamento, as amostras foram avaliadas 
por teste de Friedman, seguido de pós-teste de Dunns, considerando nível de significância de $5 \%(p<0,05)$. A presença de correlação entre os parâmetros de glicemia e temperatura nos animais submetidos à sepse (SEPSE e SEPSE+GLN) foi realizada através de análise de correlação de Spearman. As análises foram realizadas com o software GraphPad Prisma 5.0.

\section{Resultados e Discussão}

A sepse é uma doença complexa com gênese em um foco infeccioso e conseguinte disseminação sistêmica. Nesta doença ocorre o desenvolvimento de um quadro inflamatório que é seguido por uma fase hipodinâmica, fato que caracteriza a piora no quadro clínico dos pacientes, sendo que, muitas vezes, essa fase evolui para choque séptico e morte. A fase hipodinâmica é caracterizada por mudanças nos parâmetros clínicos como leucopenia4, hipotermia5, hipoglicemia6 e perda de massa muscular e peso corporal7.

Embora já existam alguns modelos experimentais que podem ser usados na avaliação da sepse, é necessário estudar um modelo experimental que mimetize de forma mais completa e adequada o quadro da doença. 0 modelo por peritonite através da administração com LPS é um modelo que utiliza um antígeno exógeno, portanto não mimetiza a infecção pela flora comensal do trato intestinal. Embora o modelo através da CLP possa representar uma infecção com as bactérias presentes nas fezes dos animais16, este modelo exige procedimento cirúrgico, o qual modifica a variável de temperatura corporal temporariamente, devido ao uso de anestesia18, o que prejudica a avaliação do próprio modelo experimental, além de influenciar em outros parâmetros fisiológicos como cardiorrespiratórios, vasculares, urinários e neurológicos 18 que são determinantes no quadro de sepse19. Assim, o modelo de administração de solução fecal do próprio animal representa vantagens em termos de mimetizar o quadro infeccioso com menores interferências metodológicas do que CLP ou LPS.

\section{Experimento 1: Dose-resposta}

Para avaliação da melhor dose que mimetizasse o quadro de sepse foram avaliados os parâmetros clínicos glicemia e temperatura corpórea. 0 protocolo experimental que melhor mimetizou a severidade do quadro de sepse foi 0 uso de solução fecal na concentração de $20 \%$, pois foi capaz de induzir e manter um quadro de hipoglicemia (Figura 1A) e hipotermia nos animais avaliados (Figura 1B).

A)

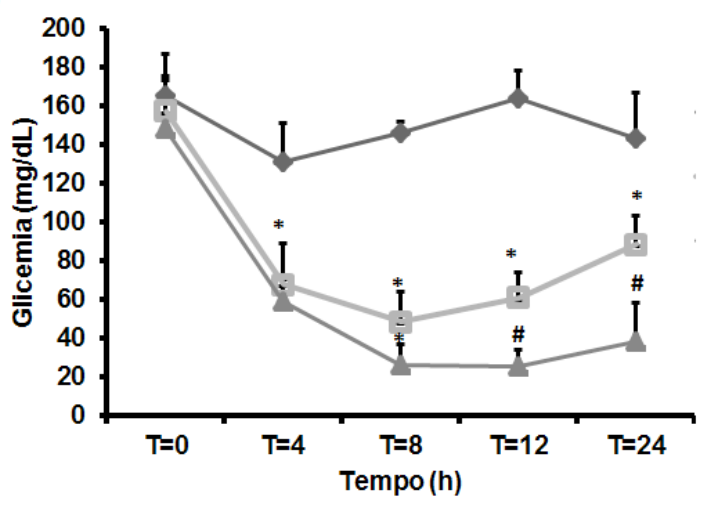

B)

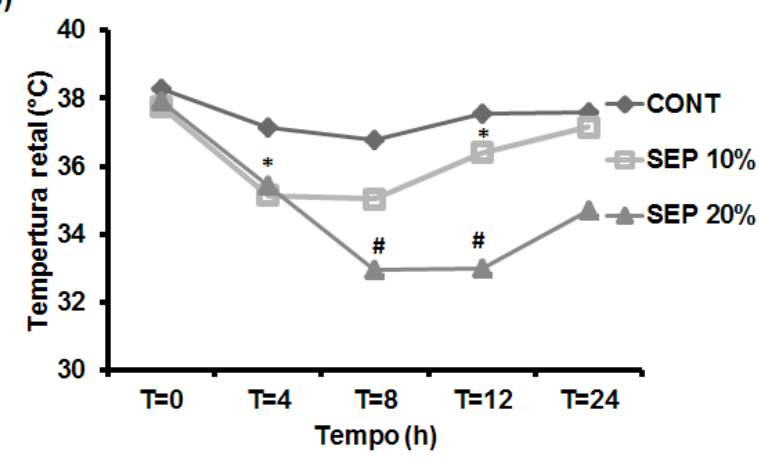

Figura 1:

Avaliação glicêmica (A) e da temperatura retal (B) 24 horas após protocolo de indução de sepse por peritonite com solução fecal $10 \%$ e $20 \%$. Teste de Kruskal-Wallis, seguido de pós-teste de Dunns, ${ }^{*}$ diferente do CONT, \# diferente do CONT e SEPSE $10 \% p<0,05$. 
A hipotermia nas primeiras 24 horas está relacionada com pior prognóstico, sendo preditor de mortalidade ${ }^{5}$, exigindo minuciosa atenção no acompanhamento do paciente ${ }^{6}$. Nosso estudo conseguiu demostrar esta piora clínica caracterizando a fase hipodinâmica da sepse, usando modelo experimental com uso de solução fecal a 20\%, levando a uma hipotermia severa de $34,7^{\circ} \mathrm{C}, 24$ horas após a indução da sepse. Este quadro se assemelha ao observado em pacientes que possuem pior prognóstico com a evolução para a choque séptico e morte 5 .

\section{Experimento 2: Avaliação do tratamento com L-glutamina}

A partir da definição da dose de $20 \%$ de solução fecal a ser utilizada para indução da sepse nos animais, teve início o experimento com intervenção de L-glutamina por gavagem na fase hipodinâmica (4 horas após a indução da sepse).

Uma das principais alterações metabólicas características da fase hipodinâmica na sepse envolve a perda de massa muscular com consequente perda de peso, sendo que os pacientes nesse quadro podem chegar a ter uma redução de até $50 \%$ da mesma7loss of the sodium-potassium gradient and to an increase in cytosolic $\mathrm{Ca}(2+$. Isto sugere que a suplementação com aminoácidos como a glutamina poderia auxiliar no tratamento da doença9nutritional support in this patient population was intended to replete substrate deficiencies secondary to stress-induced catabolism. Recognition of the influence of certain nutrients on the immune and inflammatory response of the critically ill has led to the evolution of more sophisticated nutritional strategies and concepts. Administration of immune-enhancing formulas supplemented with a combination of glutamine, arginine, omega-3 fatty acids (lu03c9-3 FA, auxiliando a manutenção da massa muscular, muito importante para a defesa do organismo no quadro de sepse20. O modelo experimental de sepse foi capaz de induzir perda de peso corporal (11\%). Na situação de catabolismo da sepse, os animais apresentaram perda de peso corporal, efeito que não foi evitado com o uso da L-glutamina (1 g/Kg/dia) (Figura 2).

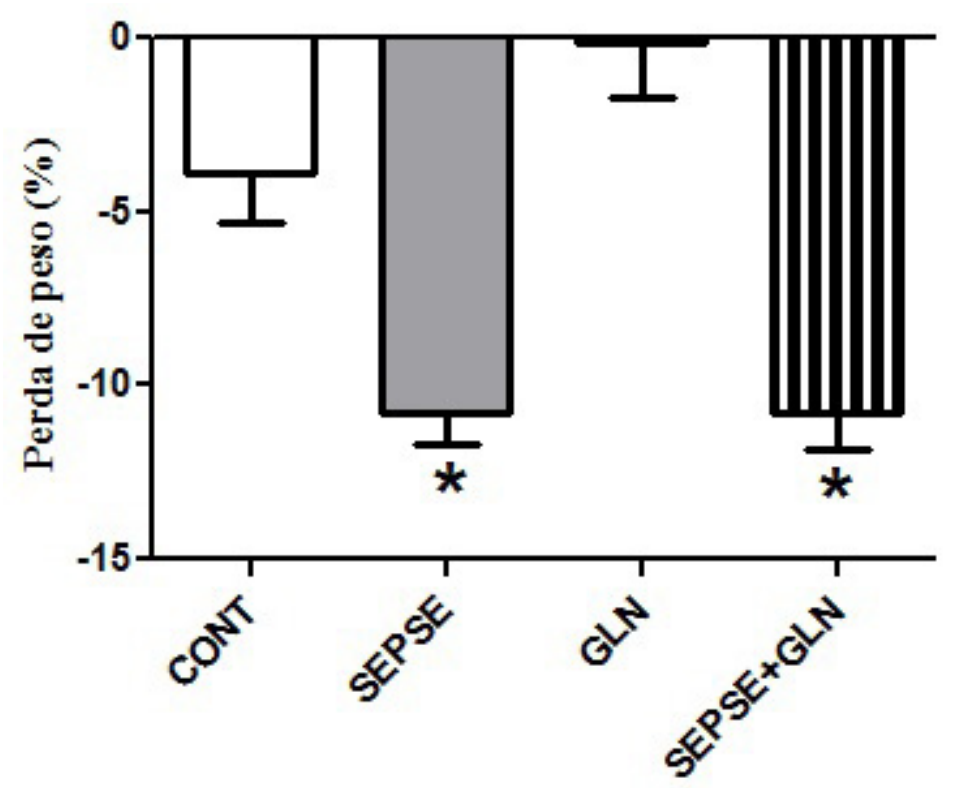

Figura 2: Perda de peso corporal (B) após 48 horas do protocolo de indução de sepse por peritonite com solução fecal 
$20 \%$, e suplementação com L-Glutamina ( $1 \mathrm{~g} / \mathrm{Kg}$ ). Teste de Kruskal-Wallis, seguido de pós-teste de Dunns ( $\mathrm{P}<0.05)$.

Resultado semelhante foi encontrado em pacientes com sepse, nos quais não foram observadas modificações no índice de massa corporal dos pacientes com a suplementação de glutamina ${ }^{13}$. Ainda, essa redução brusca de peso corporal pode promover imunossupressão, como verificado em nosso experimento explicitado pela contagem de leucócitos totais (Figura 4), o que pode estar relacionado com a diminuição da glutamina plasmática $^{13}$, motivo adicional pelo qual buscamos estudar a intervenção com glutamina.

A glicemia dos animais submetidos à sepse (SEPSE) diminuiu a partir de 4 horas, permanecendo assim até 24 horas após a indução da sepse, o que independe do tratamento com L-glutamina (Figura 3A). Quanto à temperatura corporal, o grupo SEPSE apresentou hipotermia a partir de 1 hora, permanecendo assim até 4 horas após a indução da sepse (Figura 3B) e a glutamina não modificou esse parâmetro. Foi observada ainda uma correlação positiva $(r=0,504$; $P=0,044$ ) entre a glicemia e a temperatura retal nos animais com sepse (Figura $3 C$ ), independente do tratamento com a glutamina.
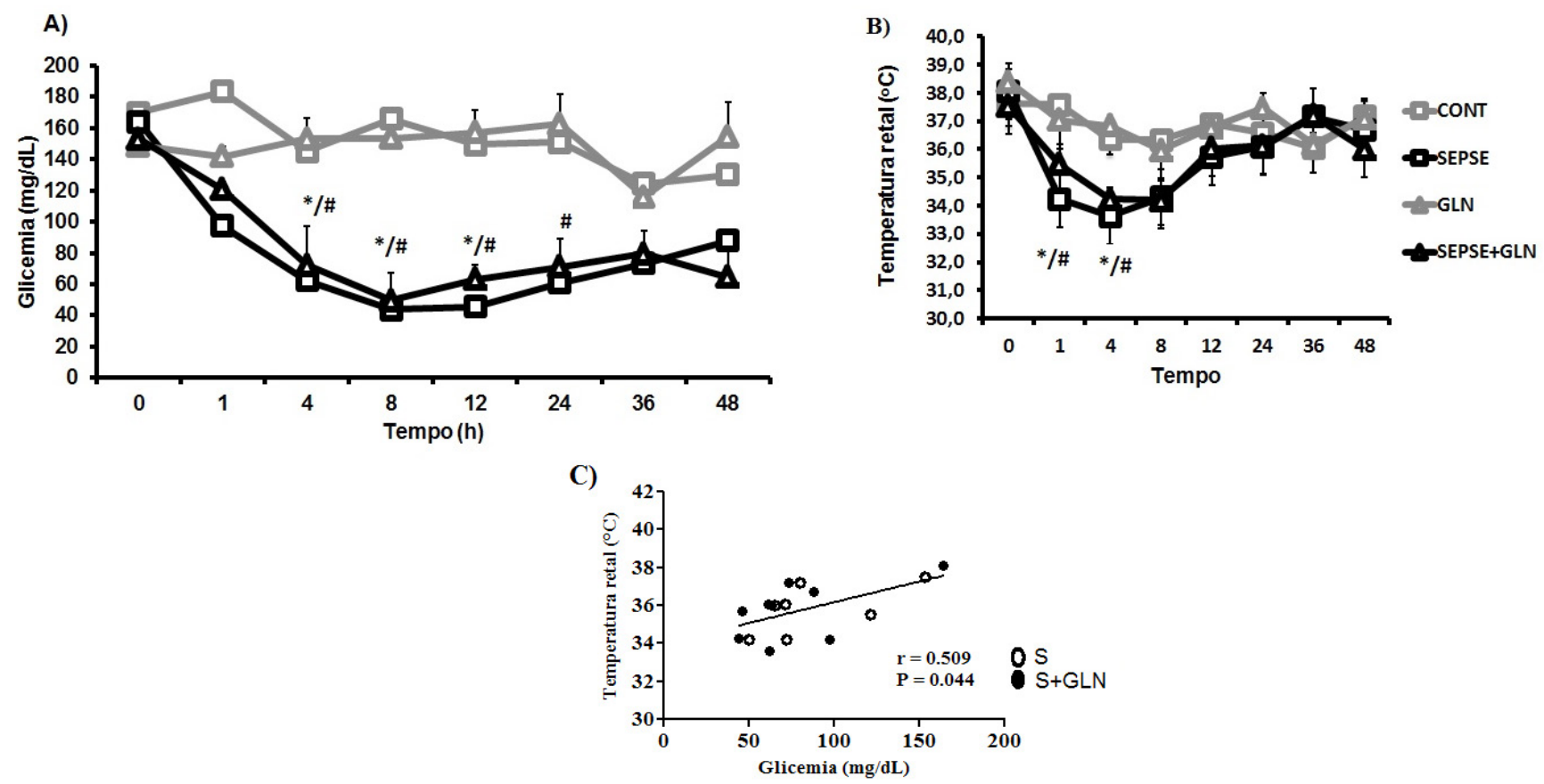

Figura 3: Glicemia (A), temperatura retal (B) em 48 horas após indução da sepse, e tratamento com L-Glutamina $(1 \mathrm{~g} / \mathrm{Kg}) .{ }^{*} P<0.05$, SEPSE e SEPSE+GLN vs respectivos tempo zero (teste de Friedman, seguido de pós-teste de Dunns). \# $P<0.05$ SEPSE e SEPSE+GLN vs respectivos Controles no mesmo tempo (teste de Kruskal-Wallis, seguido de pós-teste de Dunns). Correlação de postos de Spearman entre glicemia e temperatura (C).

Bactérias gram-negativas comensais do trato intestinal encontradas em fezes de humanos e roedores podem estar presentes na solução fecal administrada. Estas possuem a endotoxina LPS, que ocasiona diminuição na atividade da enzima hepática e renal fosfoenolpiruvato carboxiquinase (PEPCK), responsável pela gliconeogênese hepática e renal21, o que deve estar relacionado à diminuição na glicemia nos tempos 4 a 24 horas nos animais submetidos à sepse (SEPSE, SEPSE+GLN) (Figura 3A). Assim, a dose de L-glutamina administrada parece não ter sido eficaz em promover gliconeogênse hepática e renal neste estudo, o que pode estar relacionado com a diminuição das funções fisiológicas destes órgãos, ocasionadas pela suposta disfunção gerada nestes no quadro hipodinâmico da doença, mimetizado em 
nosso estudo19.

Com a diminuição da glicemia e, portanto, de substrato energético, situação na qual há diminuição no Pool energético disponível de adenosina trifosfato (ATP), sendo este, responsável por $80 \%$ da energia térmica produzida, ocorre diminuição da termogênese22. Este fato pode explicar a diminuição brusca da temperatura observada nos animais com sepse (Figura 3B). Os valores da temperatura corporal mostram-se dependentes dos valores de glicemia em nosso modelo experimental de sepse, corroborando a hipótese anterior, nos quais foram encontrados uma correlação entre ambos os parâmetros (Figura 3C).

Sendo assim, como a intervenção com L-glutamina na dose utilizada no estudo não foi capaz de promover modificações nos parâmetros de glicemia, este tratamento também não promoveu mudanças na temperatura corporal dos animais (Figura 3). Além disso, outros modelos de peritonite já demonstraram que este é capaz de promover diminuição no líquido intravascular local23, o que provoca liberação de catecolaminas levando à vasoconstrição periférica, ocasionando, assim, hipotermia24, sustentando os nossos resultados.

Ao final das 48 horas, observou-se uma diminuição ( 50\%) na contagem de leucócitos totais nos animais do grupo SEPSE em relação aos animais sadios (CONT). O tratamento com L-glutamina evitou esse efeito nos animais com sepse (SEPSE+GLN) (Figura 4).

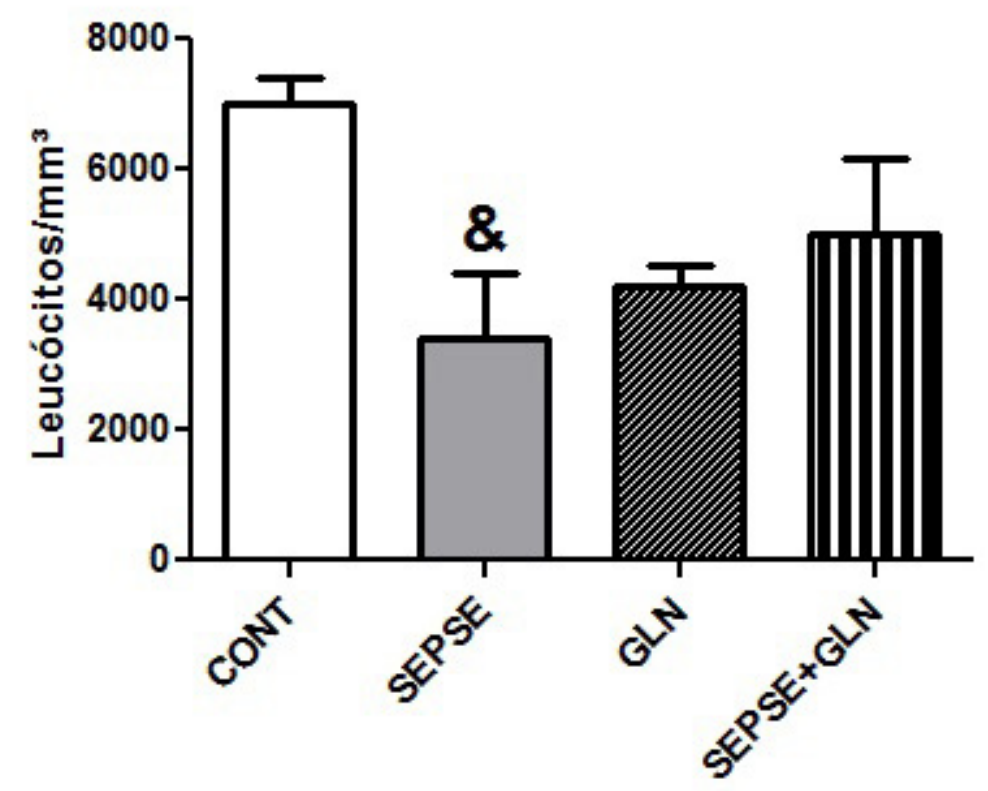

Figura 4. Avaliação da contagem de leucócitos totais após 48 horas do protocolo de indução de sepse por peritonite com solução fecal a 20\%, e suplementação com L-glutamina $(1 \mathrm{~g} / \mathrm{Kg})$. "Teste de Kruskal-Wallis, seguido de pós-teste de Dunns ( ${ }^{\&} P<0.05$, SEPSE em relação ao CONT.).

A glutamina é utilizada como fonte de energia por células do sistema imune, potencializando-as, tendo importante papel na proliferação de linfócitos, na produção de citocinas e atividade fagocitária de macrófagos e neutrófilos, 
considerado fator essencial para a defesa do organismo no quadro de sepse20.

Corroborando com o nosso estudo, já foi demonstrado que a suplementação com glutamina intravenosa foi capaz de melhorar as condições da resposta imune, aumentando quantidades de IgA plasmáticas, diminuindo a apoptose e lesão da mucosa intestinal25 e apoptose pulmonar, em modelo de sepse26, reduzindo a mortalidade27laboratory and clinical trial data have indicated a relationship between GLN-mediated protection and enhanced heat shock protein 70 (HSP70. A associação de glutamina intravenosa e enteral na sepse também diminuiu a apoptose de tecidos linfoides (baço e timo) e de citocinas pró-inflamatórias25, enquanto que a suplementação via enteral demonstra propriedades antioxidantes, alterando a expressão de proteínas citoprotetoras como as proteínas de choque térmico (Heat Shock Proteins - HSPs) 16.

Em conjunto, os resultados de nosso estudo (resumidos na tabela 1) contribuem para o aprimoramento do uso de modelos experimentais com animais para o estudo da sepse e consequentemente contribuem para o estudo de novas estratégias terapêuticas como a suplementação com glutamina. Contudo, mais estudos são necessários abordando outros parâmetros clínicos e subclínicos que envolvem a sepse para melhor compreensão do modelo experimental de indução de sepse por administração de solução fecal, assim como melhor compreensão dos efeitos do uso de glutamina.

Tabela 1. Resumo dos resultados do modelo experimental de sepse e do tratamento com glutamina

\begin{tabular}{c|c|c}
\hline Variável & $\begin{array}{c}\text { Efeito da indução de sepse por } \\
\text { administração de solução fecal } \\
\mathbf{2 0 \%}\end{array}$ & Efeito da Glutamina \\
\hline Contagem leucocitária & Leucopenia & Evitou a leucopenia \\
\hline Temperatura & hipotermia & Não evitou a hiptermia \\
\hline Glicemia & hipoglicemia & Não evitou a hipoglicemia \\
Peso corporal & Redução do peso & Não evitou a perda de peso \\
\hline
\end{tabular}

\section{Conclusão}

O estudo foi capaz de demonstrar um modelo experimental de peritonite adequado para mimetizar um quadro de sepse com sinais clínicos de hipoglicemia, hipotermia, leucopenia e perda de peso, caracterizando um quadro grave de sepse. $O$ tratamento com glutamina enteral se mostrou eficaz em evitar a diminuição da contagem de leucócitos totais, mostrando-se útil no tratamento da doença.

\section{Referências:}

1. Fleischmann C, Scherag A, Adhikari NKJ, Hartog CS, Tsaganos T, Schlattmann P, et al. Assessment of Global Incidence and Mortality of Hospital-treated Sepsis. Current Estimates and Limitations. Am J Respir Crit Care Med [Internet]. 2016 Feb 1 [cited 2017 Jun 19];193(3):259-72. Available from: http://www.ncbi.nlm.nih.gov/ pubmed/26414292

2. Barreto MFC, Dellaroza MSG, Kerbauy G, Grion CMC. Sepse em um hospital universitário : estudo prospectivo para análise de custo da hospitalização de pacientes. Rev Enferm da USP [Internet]. 2016;50(2):302-8.

Available from: http://www.scielo.br/pdf/reeusp/v50n2/pt_0080-6234-reeusp-50-02-0302.pdf

3. Doi K, Leelahavanichkul A, Yuen PST, Star RA. Animal models of sepsis and sepsis-induced kidney injury. J 
Clin Invest [Internet]. 2009 Oct 1 [cited 2017 Jun 6];119(10):2868-78. Available from: http://www.jci.org/articles/ view/39421

4. Iskander KN, Osuchowski MF, Stearns-Kurosawa DJ, Kurosawa S, Stepien D, Valentine C, et al. Sepsis: Multiple Abnormalities, Heterogeneous Responses, and Evolving Understanding. Physiol Rev [Internet]. 2013;93(3):1247-88. Available from: http://physrev.physiology.org/cgi/doi/10.1152/physrev.00037.2012

5. Tiruvoipati R, Ong K, Gangopadhyay H, Arora S, Carney I, Botha J. Hypothermia predicts mortality in critically ill elderly patients with sepsis. BMC Geriatr. 2010;10:70.

6. Park S, Kim D-G, Suh GY, Kang JG, Ju Y-S, Lee Y-J, et al. Mild hypoglycemia is independently associated with increased risk of mortality in patients with sepsis: a three-year retrospective observational study. Crit Care [Internet]. BioMed Central Ltd; 2012;16(5):R189. Available from: http://ccforum.com/content/16/5/R189

7. Wagenmakers a J. Muscle function in critically ill patients. Clin Nutr [Internet]. 2001;20(5):451-4. Available from: http://www.ncbi.nlm.nih.gov/pubmed/11534941

8. Singer M, Deutschman CS, Seymour MC, Shankar-Hari M, FFICM;, Annane D. The Third International Consensus Definitions for Sepsis and Septic Shock (Sepsis-3). 2016;315(8):801-10.

9. Santora R, Kozar RA. Molecular Mechanisms of Pharmaconutrients. J Surg Res. 2010;161(2):288-94.

10. Velasco FP da S e IT. Sepse. 1st ed. Ltda M, editor. Barueri (SP); 2007. 99-115 p.

11. Pasinato VF, Berbigier MC, Rubin B de A, Castro K, Moraes RB, Perry IDS. Terapia nutricional enteral em pacientes sépticos na unidade de terapia intensiva : adequação às diretrizes nutricionais para paciente $\cos$. Rev Bras Ter Intensiva. 2013;25(3):17-24.

.. $\quad$ Curi R. Interação do metabolismo. In: Guanabara Koogan, editor. Fisiologia humana. 2nd ed. Rio de Ja 2009. p. 778-94.

i. Tsujimoto T, Shimizu K, Hata N, Takagi T, Uejima E, Ogura H, et al. Both high and low plasma glutaminє Is predict mortality in critically ill patients. Surg Today [Internet]. Springer Japan; 2017;47(11):1331-1338. I ible from: http://link.springer.com/10.1007/s00595-017-1511-0

t. Fabiani IM, Rocha SL. Avaliação do tratamento da sepse com glutamina via enteral em ratos. Rev Col I Cir [Internet]. 2017;44(3):231-7. Available from: http://www.scielo.br/scielo.php?script=sci_arttext\&pid=? ～)$69912017000300231 \&$ Ing=pt\&tlng=pt

i. $\quad$ Yan J, Li S, Li S. The Role of the Liver in Sepsis. Int Rev Immunol [Internet]. 2014;33(6):498-510. Availi rom: http://www.tandfonline.com/doi/full/10.3109/08830185.2014.889129

16. Cruzat VF, Pantaleão LC, Donato J, de Bittencourt PIH, Tirapegui J. Oral supplementations with free and dipeptide forms of I-glutamine in endotoxemic mice: Effects on muscle glutamine-glutathione axis and heat shock proteins. J Nutr Biochem [Internet]. Elsevier Inc.; 2014;25(3):345-52. Available from: http://dx.doi.org/10.1016/j. jnutbio.2013.11.009

17. Petroianu A, Gustavo B, Carvalho M, Henrique F, Carmo O, Rocha RF. Assessment of peritoneal reinfection after fecal peritonitis in rats. Rev Col Bras Cir. 2004;31(2):90-4.

18. Souza VP De, Costa JRDR. Anestesia e neurologia: Hipotermia: Evidências Científicas. In: Anestesiologia RM de, editor. Medicina Perioperatória [Internet]. Belo Horizonte (MG); 2006. p. 539-60. Available from: http://www. saj.med.br/uploaded/file/artigos/ hipotermia.pdfgia+:+Hipotermia+:+Evidências+Científicas\#0

19. Seymour CW, Liu VX, Iwashyna TJ, Brunkhorst FM, Rea TD, Scherag A, et al. Assessment of Clinical Criteria for Sepsis: For the Third International Consensus Definitions for Sepsis and Septic Shock (Sepsis-3). Jama 
[Internet]. 2016;315(8):762-74. Available from: http://jama.jamanetwork.com/article.aspx?doi=10.1001/ jama.2016.0288\%5Cnhttp://www.ncbi.nlm.nih.gov/pubmed/26903335

20. Akagi R, Ohno M, Matsubara K, Fujimoto M, Nakai A, Inouye S. Glutamine protects intestinal barrier function of colon epithelial cells from ethanol by modulating Hsp70 expression. Pharmacology [Internet]. 2013 Jan [cited 2015 Jun 28];91(1-2):104-11. Available from: http://www.ncbi.nlm.nih.gov/pubmed/23328693

21. Caton PW, Nayuni NK, Murch $\mathrm{O}$, Corder R. Endotoxin induced hyperlactatemia and hypoglycemia is linked to decreased mitochondrial phosphoenolpyruvate carboxykinase. Life Sci [Internet]. Elsevier Inc.; 2009;84(2122):738-44. Available from: http://dx.doi.org/10.1016/j.lfs.2009.02.024

22. Douglas CR. Tratado de Fisiologia aplicada a saúde. 5th ed. Editorial R, editor. São Paulo; 2002. 1582 p.

23. Pinto CF, Watanabe M, Dezoti C, Ogata I, Vattimo F. A sepse como causa de lesão renal aguda: modelo experimental. Rev Esc Enferm USP. 2012;46:86-90.

24. Barbosa Neto JO, de Moraes MF ernand. B, Nani RS ouz., Rocha Filho JA vancin., Carmona MJ os. C. Hemostatic resuscitation in traumatic hemorrhagic shock: case report. Brazilian J Anesthesiol. 2013;63(1):99_ 102.

25. Fan J, Li G, Wu L, Tao S, Wang W, Sheng Z, et al. Parenteral glutamine supplementation in combination with enteral nutrition improves intestinal immunity in septic rats. Nutrition [Internet]. Elsevier Inc.; 2015;31(5):766-74. Available from: http://linkinghub.elsevier.com/retrieve/pii/S0899900714005462

26. Hu Y-M, Yeh C-L, Pai M-H, Lee W-Y, Yeh S-L. Glutamine Administration Modulates Lung Yס T Lymphocyte Expression in Mice With Polymicrobial Sepsis. Shock [Internet]. 2014;41(2):115-22. Available from: http:// content.wkhealth.com/linkback/openurl?sid=WKPTLP:landingpage\&an=00024382-201402000-00006

27. Singleton $\mathrm{KD}$, Wischmeyer $\mathrm{PE}$. Glutamine's protection against sepsis and lung injury is dependent on heat shock protein 70 expression. Am J Physiol Regul Integr Comp Physiol. 2007;292(5):R1839-45. 